\title{
Scheduled Virtual Topology Design Under Periodic Traffic in Transparent Optical Networks
}

\author{
Nina Skorin-Kapov \\ Dept. of Telecommunications, Faculty of Electrical \\ Engineering and Computing (FER), University of Zagreb \\ Zagreb, Croatia \\ nina.skorin-kapov@fer.hr
}

\author{
Pablo Pavon-Mariño, Belen Garcia-Manrubia, \\ Ramon Aparicio-Pardo \\ Dept. of Information and Communication Technology \\ Technical University of Cartagena, (UPCT) \\ Cartagena, Spain \\ \{pablo.pavon, belen.garcia, ramon.aparicio\}@upct.es
}

\begin{abstract}
This paper investigates offline planning and scheduling in transparent optical networks for a given periodic traffic demand. The main objective is to minimize the number of transceivers needed which make up for the main network cost. We call this problem "Scheduled Virtual Topology Design" and consider two variants: non-reconfigurable and reconfigurable equipment. We formulate both problems as exact MILPs (Mixed Integer Linear Programs). Due to their high complexity, we propose a more scalable tabu search heuristic approach, in conjunction with smaller MILP formulations for the associated subproblems. The main motivation of our research efforts is to assess the benefits of using reconfigurable equipment, realized as a reduction in the number of required transceivers. Our results show that the achieved reductions are not very significant, except for cases with large network loads and high traffic variability.
\end{abstract}

Index Terrms - All-optical networks, virtual topology design, multilayer optimization, scheduling, tabu search.

\section{INTRODUCTION}

Optical networking is evolving towards high-capacity alloptical (i.e. transparent) networks [1], [2]. In transparent networks, traffic is carried over all-optical connections, called lightpaths. A lightpath originates at a transmitter and terminates at a receiver (together referred to as transceivers), and occupies a single transmission wavelength in each traversed link. Traffic carried over a lightpath is processed electronically at the ingress and egress nodes of the lightpath (where the lightpath is said to be added or dropped, respectively), but not at intermediate transit nodes. Thus, savings with respect to electronic switching equipment is achieved.

Fig. 1 shows the typical architecture of a switching node in transparent optical networks. The core of the optical part of the node is the Wavelength Switch Fabric (WSF), which enables WDM (Wavelength Division Multiplexing) channels to be transparently switched from their input to their output ports. The WSF can be constructed as a fixed or reconfigurable component. In the former, also referred to as non-

The work described in this paper was carried out with the support of the BONE-project ("Building the Future Optical Network in Europe"), a Network of Excellence funded by the European Commission through the $7^{\text {th }}$ ICTFramework Programme, support of the MEC Spanish project TEC200767966-01/TCM CONPARTE-1 and developed in the framework of "Programa de Ayudas a Grupos de Excelencia de la Región de Murcia, de la Fundación Séneca (Plan Regional de Ciencia y Tecnología 2007/2010).” reconfigurable switching fabric, the connections between the input and output ports of the WSF are manually hard-wired and, thus, can not change on demand. In the latter, the WSF is implemented using reconfigurable optical add/drop multiplexers (R-OADM) or reconfigurable wavelength crossconnects (R-WXC), so that the connections from input to output ports can be dynamically reconfigured in tens of milliseconds.

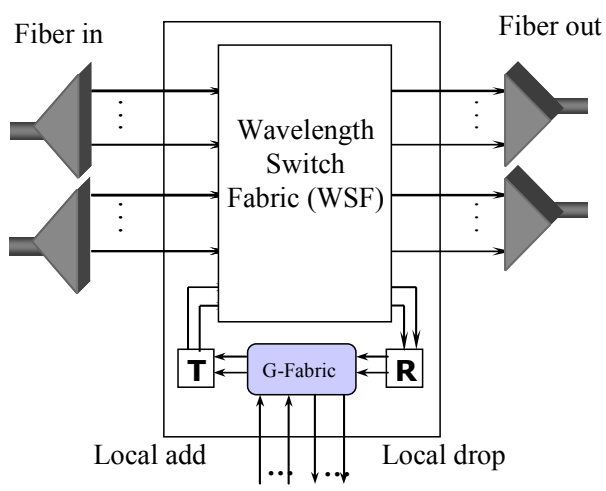

Fig. 1. The typical architecture of a switching node.

This paper investigates offline planning of transparent optical networks for a given periodic traffic demand. Namely, we consider the traffic demand to be modeled as a periodic temporal series of traffic matrices known in advance. The objective of our planning problem is to find the most costeffective set(s) of lightpaths which are capable of fully carrying the given traffic as it changes over time intervals using (non)reconfigurable equipment. A set of lightpaths established between node pairs in the network at any point in time is called a virtual topology. The virtual topology is the central element of a network multilayer problem. In the upper layer, electronic flows, i.e. traffic demands in Gbps, are routed on top of the virtual topology. Note that lightpaths are of finite capacity, and thus, determining a virtual topology capable of carrying a given traffic demand must be solved in conjunction with flow routing. In the lower layer, each lightpath in the virtual topology has to be routed over the physical topology and assigned a wavelength. This implies solving the so-called Routing and Wavelength Assignment (RWA) problem [3], [4]. 
If we focus on the consequences of the previously mentioned problems, we see that (i) defining the number of lightpaths to be established between each node pair, together with flow routing, determines the number of transceivers in the network. This number is commonly considered as the network cost figure to minimize [5]. (ii) Solving the RWA problem for the set of lightpaths which comprises the virtual topology certifies the feasibility of the network plan with respect to wavelength availability and physical impairments. However, the disparities in the network cost among different RWA solutions for the same virtual topology are not significant. Consequently, if we assume that the links in the network are equipped with a sufficient number of wavelengths and that physical-layer constraints do not apply, the associated RWA constraints can be removed from the network planning optimization problem. Such assumptions can realistically depict several network scenarios, e.g. in metro-area optical networks with an over-dimensioned fiber plant.

In this paper, we propose a set of algorithms for solving the aforementioned problem, which we denote as SVTD (Scheduled Virtual Topology Design). More formally, a SVTD instance receives a series of traffic matrices, and returns one or more virtual topology designs, together with associated flow routings. We consider two variants of the problem. In the first variant, the WSFs are assumed to be non-reconfigurable or hard-wired. Therefore, the virtual topology is constrained to be constant along time. We denote this variant as SVTD-NR (SVTD-Non-Reconfigurable). In the second variation of the problem, denoted as SVDT-R (SVTD-Reconfigurable), reconfigurable switching nodes are assumed. This means that an electro-optic transmitter in a node $N$, can at a moment $t_{1}$ be used in a lightpath between nodes $\left(N, N_{1}\right)$, and at a moment $t_{2}$ be used in a lightpath between nodes $\left(N, N_{2}\right)$. The same concept applies for opto-electronic receivers. Intuitively, fewer transceivers should be necessary to carry a given periodic traffic demand with reconfigurable equipment if peaks in traffic from node $N$ to node $N_{1}$, coincide with drops in traffic from node $N$ to other nodes. In such a case, otherwise idle transmitters can be reutilized. However, although reconfigurable equipment can yield solutions using fewer transceivers, it must be noted that such equipment is more expensive than its non-reconfigurable equivalent, creating a certain trade-off. As an example, in [5] the CAPEX of ROADM equipment was estimated to be approximately one order of magnitude higher than their non-reconfigurable counterpart.

In summary, while in the non-reconfigurable case only the flow routing can change along time, in the reconfigurable-case both the virtual topology and the flow routing can change along time. In this paper, two approaches are proposed to address the SVTD-R and SVTD-NR problems. The first approach is based on exact MILP (Mixed Integer Linear Programming) formulations, with the objective to minimize the number of transceivers required in the network, yielding optimal solutions to the problem. Clearly, both the SVTD-R and SVTD-NR problems are NP-hard, as the reduced problem with constant traffic is known to be NP-hard (integer capacity planning) [6]. Our tests indicate that solving the problem optimally using the MILP formulations can be done in reasonable time only for small network sizes and for a moderate number of time intervals. Consequently, we propose a heuristic approach for the SVTD-R/NR problems, using tabu search in conjunction with smaller MILP formulations for individual time slots within the scheduled planning problem. Tabu search is an iterative metaheuristic which guides the search procedure through the solution space using a memory structure called a tabu list. Although the MILP formulations used within the heuristics also consider NP-hard sub-problems, testing has shown that the proposed approaches are much more scalable than the exact formulation of the full problem, making them suitable for moderately-sized topologies. The effectiveness of the proposed heuristic algorithms is assessed through comparison with optimal solutions obtained by solving the MILP formulations for small topologies. For larger problem instances they are compared with proposed lower bounds on the optimal solution.

The rest of the paper is organized as follows. Section II presents the state-of-the-art of scheduled lightpaths in alloptical networks. In Section III we provide optimal MILP formulations for the SVDT-R and SVDT-NR problems. Section IV describes the tabu search heuristic approach, which includes a lower-scale MILP-based module. Section V presents the results of a case study and, finally, Section VI concludes the paper.

\section{STATE-OF-THE-ART}

The periodic nature of traffic has been confirmed with real traffic traces, such as the Abilene backbone network [7], making the expected traffic load in the network fairly predictable [8]. The first model to incorporate this phenomenon in transparent optical networks planning was the Scheduled Lightpath Demands (SLD) model from [8]. Here, the set-up and tear-down times of lightpaths are known a priori, as opposed to previous work which considered either static demands based on a single traffic matrix, or completely dynamic demands, arriving unexpectedly with random holding times. The SLD model enables more efficient utilization of resources by exploiting the temporal relationship between lightpaths. In [8], a branch and bound algorithm and tabu search heuristic were proposed for the Routing and Wavelength Assignment of a set of SLDs. An enhanced tabu search algorithm and efficient greedy algorithms for the same problem were proposed in [9]. Fault tolerant RWA was studied in [10] where the authors propose a Simulated Annealing algorithm using channel re-use and back-up multiplexing. Fault-tolerant RWA SLDs under single component failure was considered in [11]. They develop ILP formulations for the problem with dedicated and shared protection. They consider two objectives: to minimize the capacity needed to guarantee protection of all requests or maximize the number of accepted requests subject to limited capacity. In [12], the authors indicate some drawbacks in the formulations from [11], and give new ILP formulations for survivable service provisioning in networks with wavelength conversion. Their objective is to minimize the number of wavelength-links used by primary and secondary paths with guaranteed restoration in case of single failures.

A more general model, called the sliding scheduled traffic model was proposed in [13]. In this model, the set-up and 
holding times of lightpath demands are known in advance, but they are allowed to slide within a predefined window. Consequently, service provisioning consists not only of solving the RWA problem, but also scheduling demands in time subject to the sliding window constraints with the objective to minimize demand overlap. In [13], they solve the problems subsequently: first tackling scheduling using a demand time conflict reduction algorithm, and then solving RWA with two proposed approaches. Fault tolerant RWA for the sliding scheduled traffic model in networks without wavelength conversion was considered in [14]. They also propose a twophase approach: time conflict resolution followed by RWA. ILP formulations which jointly solve lightpath scheduling and RWA for the sliding scheduled traffic model are given in [15], along with a faster two-step optimization approach for larger problems.

All above mentioned approaches deal with the RWA problem of a given set of lightpath demands, whether they are fixed scheduled or can vary within a sliding window. However, the original model of SLDs proposed in [8] was motivated by the periodic nature observed in real-life traffic traces. Thus, we consider the problem of creating the set of SLDs from the periodic traffic itself, i.e. scheduling the lightpaths, which is the necessary precondition for all the above mentioned approaches. We consider a set of traffic matrices representing the estimated traffic over a series of time slots with the objective to find a set of SLDs, i.e. a set of virtual topologies over time indicating the set-up and tear-down times of the associated lightpaths, which can handle the periodic traffic in the most cost-effective manner. To the best of our knowledge, this problem has not yet been considered in the literature.

\section{MILP FORMULATIONS FOR THE SVTD-NR/R PROBLEMS}

In this section we present exact MILP formulations which search for optimal solutions to the Scheduled Virtual Topology Design (Non-)Reconfigurable (SVTD-NR/R) problems. Let $N$ be the set of nodes in the network, and $t=1, \ldots, T$ be the set of time intervals for which the traffic is defined. We denote as $|\cdot|$ the number of elements of a set. Since we are dealing with periodic traffic, we assume that the last time interval $t=T$ is followed by the first time interval $t=1$. Let $M(s, d, t), s, d \in N$, $t=1, \ldots, T$ denote the traffic demand (measured in Gbps) from node $s$ to node $d$, during time interval $t$. Let $C$ denote the lightpath capacity in Gbps. The cost of each transmitter and receiver is considered equal, and is represented by $c_{T R}$.

\section{A. SVTD-NR formulation}

The decision variables of the problem are:

- $p(i, j)=\{0,1,2, \ldots\}, i, j \in N$. The number of lightpaths from node $i$ to node $j$.

- $f(i, j, s, d, t) \geq 0, i, j, s, d \in N, t \in T$. The amount of Gbps of the traffic flow from node $s$ to node $d$ that is routed on the existing lightpaths from node $i$ to node $j$.

The problem formulation is given by (1). $\min c_{T R} \sum_{i, j \in N} p(i, j)$

subject to:

$$
\begin{aligned}
& \sum_{s, d \in N} f(i, j, s, d, t) \leq C \cdot p(i, j), \forall i, j \in N, \forall t=1, \ldots, T \\
& \sum_{j \in N} f(n, j, s, d, t)-\sum_{i \in N} f(i, n, s, d, t)=\left\{\begin{array}{l}
M(s, d, t), \text { if } n=s \\
-M(s, d, t), \text { if } n=d \\
0, \text { otherwise }
\end{array}\right. \\
& \forall n, s, d \in N, \forall t=1, \ldots, T
\end{aligned}
$$

The objective function (1a) minimizes the cost of the transceivers. Constraints (1b) represent the capacity constraints, and equations (1c) are the flow conservation constraints for the link-flow formulation.

\section{B. SVTD-R formulation}

The decision variables of the problem are:

- $p(i, j, t)=\{0,1,2, \ldots\}, \quad i, j \in N, \quad t=1, \ldots, T$. The number of lightpaths from node $i$ to node $j$, required during time interval $t$.

- $f(i, j, s, d, t) \geq 0, i, j, s, d \in N, t=1, \ldots, T$. The amount of Gbps of the traffic flow from node $s$ to node $d$ that is routed on the existing lightpaths from node $i$ to node $j$, during time interval $t$.

- $T(i)=\{0,1,2, \ldots\}, i \in N$. The number of transmitters available in node $i$.

- $R(i)=\{0,1,2, \ldots\}, i \in N$. The number of transmitters available in node $i$.

$T(i)$ and $R(i)$ represent the requirements in the number of transmitters, receivers and electronic switching capacity in node $i \in N$.

The problem formulation is given by (2).

$\min c_{T R} \sum_{i \in N}(T(i)+R(i))$

subject to:

$\sum_{s, d \in N} f(i, j, s, d, t) \leq C \cdot p(i, j, t), \forall i, j \in N, \forall t=1, \ldots, T$

$\sum_{j \in N} f(n, j, s, d, t)-\sum_{i \in N} f(i, n, s, d, t)=\left\{\begin{array}{l}M(s, d, t), \quad \text { if } n=s \\ -M(s, d, t), \text { if } n=d \\ 0, \text { otherwise }\end{array}\right.$

$\forall n, s, d \in N, t=1, \ldots, T$

$T(n) \geq \sum_{j=1}^{N} p(n, j, t), \forall n \in N, t=1, \ldots, T$

$R(n) \geq \sum_{i=1}^{N} p(i, n, t), \forall n \in N, t=1, \ldots, T$

The objective function (2a) minimizes the cost of the transmitters and receivers. Constraints $(2 \mathrm{~b})$ and $(2 \mathrm{c})$ represent the same conditions as in formulation (1). Constraints $(2 \mathrm{~d})$ and 
(2e) ensure that the number of lightpaths originating (terminating) at a given node at any time, must be below the number of transmitters (receivers) installed at that node.

\section{TABU SEARCH HEURISTIC}

Due to the high complexity of the problem, for larger instances we propose a tabu search heuristic algorithm, herein referred to as TS-SVTD. The approach iteratively solves smaller MILP formulations with various constraints on transceivers for independent time intervals in order to jump between neighboring solutions, and thus explore the solution space in a directed manner. In general, tabu search is an iterative meta-heuristic which guides the search through the solution space using a memory structure, called a tabu list, to avoid getting stuck in local optima. It does so by 'memorizing' a certain number of the most recently visited solutions, or some of their attributes, prohibiting the search to reconsider them for as long as they remain in the list. This prevents cycling between neighboring solutions around a local optimum.

A general tabu search algorithm starts with an initial current solution. Next, it explores all its neighboring solutions, and chooses the best neighboring solution (not forbidden by the tabu list) to become the new current solution in the next iteration. Potential solutions are evaluated with a fitness function. After each iteration, the tabu list and the best found solution overall, called the incumbent solution, are updated. The algorithm terminates according to a predefined termination criterion, such as the number of iterations run, the achieved solution quality, or the number of iterations without improvement.

In order to describe a specific tabu search algorithm, such as TS-SVTD, it is necessary to define the structure of a potential solution, the initial solution, the neighborhood, the fitness function, the tabu list structure, and the termination criterion.

\section{A. Structure of potential solutions and the initial solution}

A potential solution in TS-SVTD consists of $T$ virtual topologies, one for each time slot $t=1, \ldots, T$. For the nonreconfigurable case, post-processing is done to obtain a single virtual topology from the $T$ scheduled ones.

The initial solution is obtained by solving a MILP formulation for static Virtual Topology Design for each time slot separately, referred to as VTD-LT (Virtual Topology Design with Limited Transceivers). This formulation receives as input a single traffic matrix, a physical topology, and a set of upper bounds on the number of transceivers at each node. It calculates a virtual topology and its corresponding flow routing with the objective to minimize electronic switching, i.e., to minimize the number of lightpaths traversed by a unit of traffic in the network. For the initial solution, the maximum number of transceivers per node is set to infinity for each time slot. For each of the $T$ executions, the decision variables in the VTD-LT formulation are:

- $p(i, j)=\{0,1,2, \ldots\}, i, j \in N$. The number of lightpaths from node $i$ to node $j$.
- $f(i, j, s, d) \geq 0, i, j, s, d \in N$. The amount of Gbps of the traffic flow from node $s$ to node $d$ that is routed on the lightpaths from node $i$ to node $j$.

The objective function and the set of constraints are described in (4), for the problem associated with a time slot $t=1, \ldots, T$ :

$\min \sum_{i, j, s, d} f(i, j, s, d)$

subject to:

$$
\sum f(i, j, s, d) \leq C \cdot p(i, j), \forall i, j \in N
$$

$s, d \in N$

$\sum_{j \in N} f(n, j, s, d)-\sum_{i \in N} f(i, n, s, d)=\left\{\begin{array}{l}M(s, d, t), \text { if } n=s \\ -M(s, d, t), \text { if } n=d \\ 0, \text { otherwise }\end{array}\right.$

$\forall n, s, d \in N$

$\operatorname{Max} T(n) \geq \sum_{j=1}^{N} p(n, j), \forall n \in N$

$\operatorname{Max} R(n) \geq \sum_{i=1}^{N} p(i, n), \forall n \in N$

Constraints (4b) represent the capacity constraints, and equations (4c) are the flow conservation constraints. Constraints (4d) and (4e) ensure that the number of lightpaths originating (terminating) at a given node, must be below the maximum number of transmitters (receivers) at that node.

\section{B. Neighborhood}

In order to describe the neighborhood of a current solution, we first define some preliminaries.

\section{1) Preliminaries}

a) Lower bounds: We define $\mathbf{L B}_{\mathbf{T R}}(\mathrm{n})$ and $\mathbf{L} \mathbf{B}_{\mathbf{R E}}(\mathrm{n})$, for each node $\mathrm{n}$ in $\mathrm{N}$, as the lower bound on the number of transmitters and receivers, respectively, at that node. These lower bounds are described by the following expresions,

$$
\begin{aligned}
& \mathbf{L B}_{\mathbf{T R}}(n)=\left\lceil\frac{\max \left(\mathbf{T}_{\mathbf{0}}(n)\right)}{C}\right\rceil \\
& \mathbf{L B}_{\mathbf{R E}}(n)=\left\lceil\frac{\max \left(\mathbf{T}_{\mathbf{t}}(n)\right)}{C}\right\rceil
\end{aligned}
$$

where $\mathbf{T}_{\mathbf{0}}(n)$ and $\mathbf{T}_{\mathbf{t}}(n)$ is the maximum total traffic originating or terminating at node $n$, respectively, over all time intervals; while $C$ is the lightpath capacity.

b) Activity matrices: For a given solution composed of $T$ virtual topologies, we define a $(T \mathrm{x} N)$ matrix named Active Transmitters (AT) matrix. AT $(t, n)=\{0,1,2, \ldots\}$ represents the number of transmitters that are active in time slot $t$ at node $n$ in that solution. In other words, a row $t$ shows the number of active transmitters at all nodes in time slot $t$. A column $n$ shows how the number of active transmitters at node $n$ varies over time. The necessary number of transmitters per node is shown as a vector $\mathbf{T}(n)=\max (t)\{\mathbf{A T}(t, n)\}$, i.e. the maximum element in each column $n$. The total number of transmitters 
needed in the network corressponding to that solution is $T_{t o t}=\operatorname{sum}(n)\{\mathbf{T}(n)\}$. Consider the following example. Suppose there are 4 nodes and 3 time slots, i.e. $N=4, T=3$, with an Active Transmitters matrix of a potential solution as shown below.

$$
\mathbf{A T}=\left(\begin{array}{llll}
3 & 1 & 1 & 2 \\
2 & 2 & 1 & 1 \\
1 & 3 & 0 & 4
\end{array}\right)
$$

Fig. 3. An example of an AT matrix.

In this example, value $\mathbf{A T}(1,4)=2$ indicates that in the first time slot there are 2 transmitters active at node 4 . The number of necessary transmitters per node is: $\mathbf{T}(n)=\left[\begin{array}{llll}3 & 3 & 1 & 4\end{array}\right]$, while the total number of transmitters required is $T_{t o t}=11$.

We do the same for receivers to get activity matrix Active Receivers (AR), the necessary number of receivers per node $\mathbf{R}(n)$, and the total required receivers $R_{t o t}$.

c) Utilization matrices: For a given solution, we define a $(T \times N)$ matrix, which we denote as Utilization of Transmitters (UT) matrix. It is obtained from matrix AT by subtracting from each element in AT, the value of the maximal element in its column except itself. In other words,

$$
\mathbf{U T}\left(t_{i}, n_{i}\right)=\mathbf{A T}\left(t_{i}, n_{i}\right)-\max _{t \mid t \neq t_{i}}\left(\mathbf{A T}\left(t, n_{i}\right)\right)
$$

According to the above definition, the utilization matrix for the previous example is:

$$
\mathbf{U T}=\left(\begin{array}{cccc}
1 & -2 & 0 & -2 \\
-1 & -1 & 0 & -3 \\
-2 & 1 & -1 & 2
\end{array}\right)
$$

Fig. 4. An example of a UT matrix.

The positive elements in this matrix indicate the number of transmitters that are only used in a single time slot, i.e. are not very efficiently utilized. For example, UT $(3,4)=2$ indicates that in time slot 3 at node 4 , there are 2 transmitters that are only used in this time slot. Intuitively, trying to rearrange these poorly utilized transmitters may lead to better results.

We do the same for receivers from $\mathbf{A R}$ to get a matrix Utilization of Receivers (UR).

\section{2) Neigborhood reduction}

We consider neighboring solutions of a current solution to be all those where the number of transmitters or receivers per node, and the corresponding virtual topology and flow routing, are changed in only one time slot. Since there is a large number of such solutions, we propose a neighborhood reduction technique described below which reduces this set to include those solutions which we think are more likely to give good results.

Firstly, if the number of transmitters or receivers at some node in the current solution is already at its lower bound, there is no need to consider neighboring solutions which decrease the transmitters or receivers, respectively, at that node since such solutions are surely infeasible. Furthermore, recall that our main objective is to schedule the set of lightpaths and flow routings in a way which most efficiently utilizes network resources, i.e., uses the minimum number of transceivers which can carry the given periodic traffic. Thus, reducing the number of transceivers at nodes where they are highly utilized does not seem to make much sense since a reduction of these transceivers will need compensation in several time slots. Conversely, reducing a transmitter (receiver) at nodes where there are transmitters (receivers) that are used only in a single time slot can more easily be compensated for, making it more likely to find feasible solutions of higher quality. In other words, we think eliminating poorly utilized transceivers where feasible should yield better results.

Consequently, we perform neighborhood reduction as follows. For each node, we choose one time slot with poorly utilized transmitters and one time slot with poorly utilized receivers, except for nodes forbidden by the tabu list (as described in Section IV.D). ${ }^{1}$ We denote these 'candidates' as triples in the form: ( $\mathrm{n}, \mathrm{t}$, ' $\mathrm{tr}$ '/ 're'), each corresponding to one neighboring solution. The number of candidates (or neighbors) is thus $2 \cdot \mathrm{n}$ - (the size of the tabu list). Note that candidates cannot include nodes at time slots for which the lower bound on transmitters/receivers is reached.

To choose the set of candidates ( $\mathrm{n}, \mathrm{t}$, 'tr') with respect to transmitters, we consider only strictly positive, i.e. poorly utilized, elements in $\mathbf{U T}(t, n)$, for which $\mathbf{A T}(t, n)>\mathbf{T} \_\mathbf{L B}(n)$. For each node $n$, we choose one such element at random which is not forbidden by the tabu list. If there are no positive elements in $\mathbf{U T}(t, n)$, we choose at random a time slot corresponding to one of the elements with a value of zero (there is always at least one such element). For each obtained candidate (n, $t$, ' $\operatorname{tr}$ '), we run the VTD-LT formulation for time slot $t$ (i.e. with the traffic matrix at time $t$ ) but bound the maximum number of transmitters at node $n$ to $\mathbf{A T}(t, n)-1$. Receivers at node $n$, along with transmitters and receivers at all other nodes, are bounded to their maximal value along time in the current solution. The new virtual topology obtained by solving the VTD-LT formulation replaces the virtual topology at corresponding time slot $t$ in the current solution, giving the new neighboring solution. The same is done to obtain neighbors from candidates (n, t, 're') with respect to receivers, chosen analogously from non-negative elements of $\mathbf{U R}(t, n)$ for which $\mathbf{A R}(t, n)>\mathbf{L B}_{\mathbf{R E}}(n)$

In our example, assuming no violation of the lower bound and tabu list constraints, we would have four neighbors with respect to transmitters obtained from candidates $\left(1,1\right.$, ' $\left.\operatorname{rr}^{\prime}\right),(2$, 3 , 'tr' $),(3,1$ or 2 , 'tr') and (4, 3, 'tr'), which correspond to elements UT( 1,1$)$, UT( 3,2$)$, UT( 1,3$)$ or UT(2,3), and UT $(3,4)$, respectively. Four more neighbors could be to obtained from candidates obtained from poorly utilized receivers. If all neighboring solutions in the reduced neighborhood are infeasible in an iteration, the neighborhood is increased to $2 \cdot t \cdot n$ where candidates correspond to all nodes and all time slots for both transmitters and receivers, and with no constraints imposed by the tabu list.

1 The same time slot can be chosen for both poorly utilized transmitters and receivers. 


\section{Fitness function}

To choose the best neighboring solution to become the new current solution in the next iteration, we define two different fitness functions for two variations of the algorithm.

a) Non-reconfiguration equipment: This fitness function has the objective to minimize the number of transceivers per node in the non-reconfigurable case where transmitters and receivers at the same node cannot be used for different lightpaths in different time intervals. Consequently, the number of transceivers per node corresponds to the maximal number of different lightpaths originating and terminating at that node over all time intervals. It follows that the fitness function is:

$$
\sum_{n=1}^{N} \sum_{m=1, m \neq n}^{N} \max _{t} \boldsymbol{L p}(n, m, t)
$$

where $\boldsymbol{L} \boldsymbol{p}(n, m, t)$ is the number of lightpaths established between the node $n$ and the node $m$ at the time slot $t$.

b) Reconfigurable equipment: This fitness function aimes to minimize the number of transceivers used assuming reconfigurable equipment, i.e., the same transceivers can be used for different lightpaths as long as they are in different time slots. It is:

$$
\sum_{n=1}^{N} \max _{t} \sum_{m, m \neq n}^{N} \boldsymbol{L p}(n, m, t)
$$

\section{Tabu list structure and termination criterion}

Each entry in the tabu list is a pair ( $n$, 'tr'/'re') representing the node $n \in N$ at which either transmitters (tr) or receivers (re) were reduced in order to get the new current solution from the
TABLE I

\begin{tabular}{c|c|c|c|c|c}
\multicolumn{6}{c}{5 Node NETWORK TRAFIC MATRIX IN GBPS } \\
\hline & 0 & 1 & 2 & 3 & 4 \\
\hline 0 & & 88.8 & 30.6 & 51.0 & 77.8 \\
\hline 1 & 285.6 & & 166.8 & 278.6 & 425.2 \\
\hline 2 & 98.0 & 166.8 & & 95.6 & 146.0 \\
\hline 3 & 163.8 & 278.6 & 95.6 & & 243.8 \\
\hline 4 & 250.0 & 425.2 & 146.0 & 243.8 & \\
\hline
\end{tabular}

previous one. For as long as this pair remains in the tabu list, the transmitters/receivers at node $n$ cannot be further reduced. The tabu list is realized as a FIFO (First In First Out) queue of finite size, updated after each iteration. The termination criterion for the algorithm is defined by the maximum number of iterations which can be run without improvement of the incumbent solution.

\section{E. Postprocessing}

For the non-reconfigurable case, a post-processing step is necessary since the final solution must be in the form of a single virtual topology, while the tabu search algorithm gives a set of $T$ virtual topologies. To achieve a single virtual topology $T$, which can handle all the traffic over time from the obtained solution, we establish $\boldsymbol{L} \boldsymbol{p}(n, m)$ lightpaths between nodes $n$ and $m$, where $\boldsymbol{L} \boldsymbol{p}(n . m)=\max ($ along time $)\{\boldsymbol{L} \boldsymbol{p}(n, m, t)\}, n, m$ in $N, t$ in $T$, from the solution given by TS-SVTD. To assign individual traffic flow routings for each time slot over the obtained static virtual topology, we use a multi-commodity flow LP (Linear Programming) formulation yielding optimal solutions.

TABLE II

\begin{tabular}{|c|c|c|c|c|c|c|c|c|c|c|c|c|c|c|c|c|c|c|}
\hline & 0 & 1 & 2 & 3 & 4 & 5 & 6 & 7 & 8 & 9 & 10 & 11 & 12 & 13 & 14 & 15 & 16 & 17 \\
\hline 0 & & 8.5 & 8.5 & 17.0 & 76.5 & 8.5 & 8.5 & 42.5 & 8.5 & 8.5 & 8.5 & 8.5 & 8.5 & 8.5 & 25.5 & 17.0 & 8.5 & 8.5 \\
\hline 1 & 8.5 & & 8.5 & 51.0 & 68.0 & 8.5 & 8.5 & 17.0 & 8.5 & 34.0 & 8.5 & 8.5 & 8.5 & 8.5 & 25.5 & 34.0 & 8.5 & 8.5 \\
\hline 2 & 8.5 & 8.5 & & 8.5 & 25.5 & 8.5 & 8.5 & 8.5 & 8.5 & 8.5 & 8.5 & 8.5 & 8.5 & 8.5 & 8.5 & 8.5 & 8.5 & 8.5 \\
\hline 3 & 17.0 & 51.0 & 8.5 & & 93.5 & 8.5 & 8.5 & 42.5 & 8.5 & 42.5 & 8.5 & 8.5 & 34.0 & 17.0 & 51.0 & 85.0 & 8.5 & 8.5 \\
\hline 4 & 76.5 & 68.0 & 25.5 & 93.5 & & 17.0 & 8.5 & 76.5 & 17.0 & 68.0 & 17.0 & 8.5 & 34.0 & 51.0 & 93.5 & 68.0 & 17.0 & 17.0 \\
\hline 5 & 8.5 & 8.5 & 8.5 & 8.5 & 17.0 & & 8.5 & 17.0 & 8.5 & 8.5 & 8.5 & 8.5 & 8.5 & 8.5 & 8.5 & 8.5 & 8.5 & 8.5 \\
\hline 6 & 8.5 & 8.5 & 8.5 & 8.5 & 8.5 & 8.5 & & 8.5 & 8.5 & 8.5 & 8.5 & 8.5 & 8.5 & 8.5 & 8.5 & 8.5 & 8.5 & 8.5 \\
\hline 7 & 42.5 & 17.0 & 8.5 & 42.5 & 76.5 & 17.0 & 8.5 & & 8.5 & 17.0 & 8.5 & 8.5 & 25.5 & 8.5 & 51.0 & 25.5 & 8.5 & 8.5 \\
\hline 8 & 8.5 & 8.5 & 8.5 & 8.5 & 17.0 & 8.5 & 8.5 & 8.5 & & 8.5 & 8.5 & 8.5 & 8.5 & 8.5 & 8.5 & 8.5 & 8.5 & 8.5 \\
\hline 9 & 8.5 & 34.0 & 8.5 & 42.5 & 68.0 & 8.5 & 8.5 & 17.0 & 8.5 & & 8.5 & 8.5 & 17.0 & 8.5 & 25.5 & 42.5 & 8.5 & 8.5 \\
\hline 10 & 8.5 & 8.5 & 8.5 & 8.5 & 17.0 & 8.5 & 8.5 & 8.5 & 8.5 & 8.5 & & 8.5 & 8.5 & 8.5 & 8.5 & 8.5 & 8.5 & 8.5 \\
\hline 11 & 8.5 & 8.5 & 8.5 & 8.5 & 8.5 & 8.5 & 8.5 & 8.5 & 8.5 & 8.5 & 8.5 & & 17.0 & 8.5 & 8.5 & 8.5 & 8.5 & 8.5 \\
\hline 12 & 8.5 & 8.5 & 8.5 & 34.0 & 34.0 & 8.5 & 8.5 & 25.5 & 8.5 & 17.0 & 8.5 & 17.5 & & 8.5 & 17.0 & 8.5 & 8.5 & 0.0 \\
\hline 13 & 8.5 & 8.5 & 8.5 & 17.0 & 51.0 & 8.5 & 8.5 & 8.5 & 8.5 & 8.5 & 8.5 & 8.5 & 8.5 & & 8.5 & 8.5 & 8.5 & 8.5 \\
\hline 14 & 25.5 & 25.5 & 8.5 & 51.0 & 93.5 & 8.5 & 8.5 & 51.0 & 8.5 & 25.5 & 8.5 & 8.5 & 17.0 & 8.5 & & 25.5 & 8.5 & 8.5 \\
\hline 16 & 8.5 & 8.5 & 8.5 & 8.5 & 17.0 & 8.5 & 8.5 & 8.5 & 8.5 & 8.5 & 8.5 & 8.5 & 8.5 & 8.5 & 8.5 & 8.5 & & 8.5 \\
\hline 17 & 8.5 & 8.5 & 8.5 & 8.5 & 17.0 & 8.5 & 8.5 & 8.5 & 8.5 & 8.5 & 8.5 & 8.5 & 0.0 & 8.5 & 8.5 & 8.5 & 8.5 & \\
\hline
\end{tabular}

18 NODE NETWORK TRAFFIC MATRIX IN GBPS 
TABLE III

Total Number of TRANSCEIVERS NEEDED FOR THE 5-NODE NETWORK

\begin{tabular}{c|c|c|c|c|c|c}
\hline \multirow{2}{*}{$\boldsymbol{n f}$} & \multirow{2}{*}{$\boldsymbol{R}$} & \multirow{2}{*}{$\begin{array}{c}\text { Lower } \\
\text { Bound }\end{array}$} & \multicolumn{2}{c|}{ Reconfigurable } & \multicolumn{2}{c}{$\begin{array}{c}\text { Non- } \\
\text { Reconfigurable }\end{array}$} \\
\cline { 4 - 7 } & & & $\begin{array}{c}\text { SVTD } \\
\text { MILP-R }\end{array}$ & $\begin{array}{c}\text { TS - } \\
\text { SVTD }\end{array}$ & $\begin{array}{c}\text { SVTD } \\
\text { MILP- } \\
\text { NR }\end{array}$ & $\begin{array}{c}\text { TS - } \\
\text { SVTD }\end{array}$ \\
\hline \multirow{2}{*}{500} & $10 \%$ & 109 & 110 & 120 & 110 & 122 \\
Gbps & $20 \%$ & 116 & 116 & 125 & 118 & 132 \\
& $50 \%$ & 123 & 123 & 131 & 126 & 154 \\
\hline \multirow{2}{*}{1000} & $10 \%$ & 214 & 214 & 222 & 216 & 228 \\
Gbps & $20 \%$ & 223 & 223 & 228 & 226 & 248 \\
& $50 \%$ & 259 & 259 & 266 & 262 & 304 \\
\hline \multirow{2}{*}{2000} & $10 \%$ & 419 & 419 & 423 & 422 & 446 \\
Gbps & $20 \%$ & 444 & 444 & 453 & 446 & 482 \\
& $50 \%$ & 518 & 518 & 528 & 524 & 572 \\
\hline
\end{tabular}

\section{RESUlTS}

We studied the performance of the proposed algorithms for two network sizes: 5 and 18 nodes. The 5-node network was based on a reduced carrier network and the 18-nodes on the EON network [16]. However, the particular physical topology is not relevant for our problem. We assume that sufficient resources exist in the physical topology (i.e. sufficient number of wavelengths in the physical links) so that every virtual topology design has a feasible RWA solution. Consequently, our algorithms take as input only the number of nodes in the network. For the smaller network, the exact MILP formulations (SVTD-NR/R) were run and compared with the results obtained by the TS-SVTD algorithm to asses efficiency of the heuristic approach. For the medium-sized 18-node scenario, only variations of TS-SVTD were tested, given the intractability of the pure MILP approach. The algorithms were implemented using the MatPlanWDM tool [17], which interfaces to a TOMLAB/CPLEX library [18] used to solve MILP problems. For both scenarios, two cases were considered: (i) a network with non-reconfigurable equipment, (ii) a network with reconfigurable equipment.

A series of traffic matrices were synthesized to feed the planning algorithms. Each traffic matrix series was composed of 24 traffic matrices, one for each hour of the day (i.e., $t=1, \ldots, 24)$, obtained from a single base traffic matrix and an activity function. The activity function describes fluctuations in traffic over the course of a typical day. The traffic synthesis model is described by:

$$
\mathbf{T}(i, j, t)=\mathbf{T}_{\text {Base }} \cdot \frac{n f}{\sum_{i, j=1}^{N} \mathbf{T}_{\text {Base }}(i, j)} \cdot \operatorname{activity}(t) \cdot r f(R)
$$

$\mathbf{T}_{\text {Base }}$ is a base traffic matrix calculated for each scenario. In the 5-node network, the base traffic matrix is shown in Table I and was obtained from traffic forecast studies for a national optical backbone (measured inGbps). In the 18-node network, the base traffic matrix was obtained from [16], and normalized to match a total offered traffic of 5 Tbps (Table II).

The normalization factor $(n f)$ is selected to make the total offered traffic in the base traffic matrix $\left(\mathbf{T}_{\text {Tot }}=\mathbf{T}_{\text {Base }} * \mathbf{T}_{\text {Norm }}\right.$, where $\mathbf{T}_{\text {Norm }}$ is the quotient in expression (10)) match a desired
TABLE IV

TOtAL NuMBER OF TRANSCEIVERS NEEDED FOR THE 18-NODE NETWORK

\begin{tabular}{c|c|c|c|c}
\hline $\boldsymbol{n} \boldsymbol{f}$ & $\boldsymbol{R}$ & $\begin{array}{c}\text { Lower } \\
\text { Bound }\end{array}$ & $\begin{array}{c}\text { Reconfigurable } \\
\text { TS-SVTD }\end{array}$ & $\begin{array}{c}\text { Non- } \\
\text { Reconfigurable } \\
\text { TS-SVTD }\end{array}$ \\
\hline \multirow{2}{*}{1500} & $10 \%$ & 322 & 720 & 724 \\
Gbps & $20 \%$ & 331 & 720 & 728 \\
& $50 \%$ & 359 & 733 & 758 \\
\hline \multirow{2}{*}{3000} & $10 \%$ & 631 & 912 & 946 \\
Gbps & $20 \%$ & 650 & 918 & 962 \\
& $50 \%$ & 689 & 947 & 1042 \\
\hline \multirow{2}{*}{6000} & $10 \%$ & 1248 & 1522 & 1786 \\
Gbps & $20 \%$ & 1283 & 1517 & 1824 \\
& $50 \%$ & 1371 & 1566 & 1984 \\
\hline
\end{tabular}

value. The total traffic values tested were $\mathbf{T}_{\text {Tot }}=\{500,1000,2000\}$ Gbps for the 5-node network, and $\mathbf{T}_{\text {Tot }}=\{1500,3000,6000\}$ Gbps for the 18-node network.

The factor activity $(t)$ represents the activity function of the network, i.e. it is a function which intends to capture the effect of traffic intensity variation over the day. Our intensity variation model is described by equation (11), based on the intensity model presented in [19].

$\operatorname{activity}(t)=\left\{\begin{array}{l}0.1 \text { if } t \in[1,6] \\ 1-0.9 \cdot\left(\cos \left(\frac{\bmod (t, T)-6}{18} \cdot \pi\right)\right)^{10} \text { otherwise }\end{array}\right.$

where $t=1, \ldots, 24, T$ is the total number of time intervals

The function $r f(R)$ computes a matrix where each coordinate is uniformly calculated between $[1-R, 1+R]$, where $R$ is a value between 0 and 1 . We call $R$ random factor. The objective of the $r f$ factor is to capture a randomness effect in the traffic intensity, which may control the predictability of the traffic series. The random factors used are $R=\{0.1,0.2,0.5\}$, corresponding to low, medium and high random variation scenarios.

The parameters of the tabu search algorithm were defined as follows. The length of the tabu list was set to three for the 5node scenario and nine for the 18-node scenario. The termination criterion was set to 100 iterations without improvement.

Table III shows the results obtained for the 5-node network. We evaluated the solutions obtained by solving all proposed variants of the MILP formulation and tabu search algorithm in terms of the total number of transceivers needed. The lower bounds on the number of transceivers as calculated by expression (5) are also included to asses their efficiency. Note that the same lower bounds apply to the reconfigurable and non-reconfigurable cases. Results show that the resources planned by the optimal MILP formulation are equal to the computed lower bound in almost all cases, validating their accuracy in this scenario. Slight variations are found in the non-reconfigurable case. As expected, the number of transceivers planned is higher for series of traffic matrices with 
a higher traffic variability factor $R$. This increase is more significant at higher loads. The tabu search scheme allocates only a slightly higher amount of resources when compared to the optimal MILP solution indicating its efficiency. The extraresources planned are in the order of $10 \%$ in the nonreconfigurable case, and 5\% in the reconfigurable case.

As already mentioned, the results for the 18-node network were obtained only for the tabu-search scheme due to the intractability of the exact MILP formulation. These results are shown in Table IV. The MILP module used to calculate neighbors within the tabu search approach (i.e., the VTD-LT formulation) can be solved in reasonable time for the examples tested. Namely, VTD-LT completion time was in the order of 1 to 5 seconds. Consequently, a heuristic-based solution of this sub-problem was not attempted in this paper, but we plan to investigate such variations for large-scale networks for future work.

The solutions obtained for the 18-node network at lower loads show a large gap in the number of transceivers between the lower bounds and the solutions obtained by TS-VTD. Namely, the solutions found double the resources predicted by the lower bounds. At higher loads this gap is much smaller, i.e., in the order of the $25 \%$. The planned number of transceivers does not seem to be significantly affected by the variability factor $R$, although in the non-reconfigurable case we can see slight variations. If we compare the number of transceivers needed for the non-reconfigurable and reconfigurable cases, we observe that the difference is not significant at lower loads, increasing to some extent at higher loads. However, the maximal reduction in the number of transceivers obtained (i.e., the case for the 18- node network with maximal traffic load and variability) was only $21.07 \%$. The average reduction over all cases was $5.51 \%$. Considering the significant higher price of reconfigurable equipment, our results indicate that reconfiguration may not be cost-effective, even for cases when traffic is highly variable.

\section{CONCLUSIONS}

In this paper, we propose a new planning problem in optical networks, which we call "Scheduled Virtual Topology Design", to schedule lightpaths according to given periodic traffic. Two variants are proposed, assuming nonreconfigurable and reconfigurable equipment, with the objective to minimize the number of transceivers needed to handle the given traffic. We formulate both variations of the problem as exact MILP (Mixed Integer Linear Programs) and propose analytical lower bounds. For small networks, the MILP solutions are very close to the lower bound. Due to the intractability of the pure MILP, we propose a hybrid tabu search heuristic algorithm which obtains suboptimal solutions. The approach iteratively solves smaller MILP formulations within the tabu search algorithm which optimize the network plan in a single time slot. This approach has shown to be suitable for the medium-sized topology tested. Our results indicate that the reduction with respect to the transceivers obtained with reconfigurable equipment is not significant, except in the case of larger network loads associated with high traffic variability. However, even in these cases, the reduction is not dramatic and considering the higher cost of such equipment, does not seem to be cost-effective. For future work we will develop a pure heuristic approach for large problems and perform a detailed cost-benefit analysis of using reconfigurable components.

\section{REFERENCES}

[1] J. Berthold, A. A. M. Saleh, L. Blair, and J. M. Simmons, "Optical Networking: Past, Present, and Future," Journal of Lightwave Technology, vol. 26, no. 9, pp.1104-1118, May 2008.

[2] S. Sygletos, I. Tomkos, and J. Leuthold, "Technological challenges on the road toward transparent networking," Journal of Optical Networking, vol. 7, no. 4, pp. 321-350, Apr. 2008.

[3] H. Zang, J. P. Jue, and B. Mukherjee, "A Review of Routing and Wavelength Assignment Approaches for Wavelength-Routed Optical WDM Networks," Optical Network Magazine, pp. 47-59, Jan. 2000.

[4] R. Ramaswami, and K. N. Sivarajan, "Routing and Wavelength assignment in all-Optical networks", IEEE/ACM Transactions on Networking, vol. 5, no. 3, pp.489-500, Oct. 1995.

[5] NOBEL phase 2 -Deliverable D 2.4. "Migration Guidelines with Economic Assessment and New Business Opportunities Generated by NOBEL phase 2" [Online]. Available: http://www.istnobel.org/Nobel2/imatges/D2.4 final\%20version.pdf.

[6] B. Mukherjee, D. Banerjee, S. Ramamurthy, A. Mukherjee, "Some Principles for Designing a Wide-Area WDM Optical Network," IEEE/ACM Transactions on Networking, Vol. 4, No. 5, pp. 684-696, October 1996.

[7] TOTEM Project: Toolbox for Traffic Engineering Methods [Online]. Available: http://totem.run.montefiore.ulg.ac.be/datatools.html

[8] J. Kuri, N. Puech, M. Gagnaire, E. Dotaro, and R. Douville, "Routing and wavelength assignment of scheduled lightpath demands," IEEE J. Select. Areas Commun., vol. 21, pp. 1231-1240, Oct. 2003.

[9] N. Skorin-Kapov, "Heuristic algorithms for the routing and wavelength assignment of scheduled lightpath demands in optical networks," IEEE J. Select. Areas Commun., vol. 24, pp. 2-15, 2006.

[10] J. Kuri, N. Puech, and M. Gagnaire, "Diverse routing of scheduled lightpath demands in an optical transport network," in Proc. Design of Reliable Communication Networks (DCRN2003), pp. 69-76, Oct. 2003.

[11] C. V. Saradhi, L. K. Wei, and M. Gurusamy, "Provisioning fault-tolerant scheduled lightpath demands in WDM mesh networks", in Proc. of BroadNets 2004, San Jose, Cal. USA, pp. 150-159, Oct. 2004.

[12] B. Wang, T. Li, C. Xin, X. Zhang, "On survivable service provisioning in WDM networks under a scheduled traffic model", in Proc. of Globecom 2005, St. Louis, MO, USA, pp. 1900-1904, Nov.-Dec. 2005.

[13] B.,Wang, T. Li, X. Luo, Y. Fan, and C. Xin, ”On service provisioning under a scheduled traffic model in reconfigurable WDM optical networks“ in Proc. Of BroadNets 2005, Boston, USA, pp 13-22, Oct. 2005.

[14] C. V. Saradhi, M. Gurusamy,and R. Piesiewicz, "Routing fault-tolerant sliding scheduled traffic in WDM optical mesh networks" in Proc. of BroadNets 2008, London, UK, pp.197-202, Sept. 2008.

[15] A. Jaekel, Y. Chen, "Resource provisioning for survivable WDM networks under a sliding cheduled traffic model", Optical Switching and Networking, Vol. 6, pp. 44-54, 2009.

[16] L. Wuttisittikulkij, M.J. O'Mahony, "Design of a WDM network using a multiple ring approach", in Proc. IEEE Global Telecommunications Conference, Phoenix, AZ, 1997, vol. 1, pp. 551-555.

[17] P. Pavon-Mariño, R. Aparicio-Pardo, G. Moreno-Muñoz, J. GarciaHaro, J. Veiga-Gontan, "MatPlanWDM: An educational tool for network planning in wavelength-routing networks", Proc. IFIP Int. Conf. Optical Network Design and Modelling, Athens, Greece, 2007, pp. 58-67.

[18] TOMLAB Optimization. [Online]. Available: http://tomopt.com/

[19] J. Milbrandt, M. Menth, S. Kopf, 2005 "Adaptive Bandwidth Allocation: Impact of Traffic Demand Models for Wide Area Networks", in 19th International Teletraffic Congress (ITC19), Beijing, China, 2005. 